\title{
THE MASS STAINING OF PARAFFIN SECTIONS BEFORE THE REMOVAL OF WAX
}

\author{
BY \\ C. F. A. CULLING \\ From the Westminster School of Medicine
}

(RECEIVED FOR PUBLICATION, JANUARY 7, 1949)

The problem of producing large numbers of sections for teaching purposes and staining them by haematoxylin and eosin has become acute in the post-war years, due to the shortage both of trained technicians and of reagents, especially ethyl alcohol. Methods previously used have suffered from the disadvantages of being too long, or wasteful of reagents, and it was with these factors in mind that the following method was evolved.

Robb-Smith (1937) showed that free paraffin sections could be impregnated with silver. Mayer also showed that methyl violet would stain amyloid in paraffin sections that had not been de-waxed. This principle has been adapted for the mass staining of paraffin sections before the removal of wax.

\section{Apparatus}

An M.S.E. rotary-type microtome is used in this department as it is thought to give better ribbons of sections. Half of a 7-in. Petri dish or a developingdish of similar size has been found sufficient for $\mathbf{6 0}$ sections of average size $(2 \mathrm{~cm} . \times 1 \mathrm{~cm}$.). Four washbottles with rubber and glass attachments may be prepared from empty wide-mouthed bottles, rubber-andglass tubing, and rubber bungs. A glass filter pump is at present used, positive pressure being obtained by blowing. Dissecting needles and staining solutions are also needed.

\section{Method}

Some sixty to seventy sections are cut in the usual manner. The use of ice on the surface of the paraffin block before and during cutting gives flatter sections.

The dish is half-filled with water at a temperature of approximately $45^{\circ} \mathrm{C}$. when using $54^{\circ}$ C.M.P. wax (see Appendix).

Ribbons are cut to a convenient length to give halfinch clearance each end when flattened in the dish. The method of removal and replacement of solutions follows the same pattern throughout and is as follows.

The glass tube connected to the wash-bottle (see Appendix, 2) is passed down the side of the dish to the bottom, and negative pressure is applied. While the surface of the water is falling, the ribbons are kept free of the side of the dish by needles; otherwise they become attached. If this should happen, they are' easily freed by turning off the filter-pump and tilt-. ing the dish gently towards the attached section. The last few millilitres are removed by tilting the dish towards the suction tube. This is done slowly, as any rapid movement will cause telescoping of sections. Pressure is reduced towards the end of this operation.

Solutions are run in by a reverse procedure, positive pressure being applied to the wash-bottle, either with a pump or by blowing to start the stream, when the remainder will siphon over if the bottle is lifted. Only enough solution to float the sections is required.

The following solutions and times have been found to give the best results with haematoxylin and eosin:

1. Tap water at $45^{\circ} \mathrm{C}$. to flatten sections. Heated distilled water may be substituted for this, as it combines stages 1 and 2 , but time is gained by this procedure only if hot water is not readily available.

2. Distilled water-2/minutes.

3. Haematoxylin-40 minutes (see Appendix, 3).

4. Distilled water -2 minutes.

5. Acid alcohol (1 per cent in 95 per cent alcohol) -1 minute (see Appendix, 4).

6. Ammonia water ( 0.1 per cent in distilled water) -until sections are blue.

7. Tap water -2 minutes.

8. Eosin ( 1 per cent in distilled water)-20 minutes.

9. Tap water (cold).

Sections are left as short a time as possible on tap water after stage 8 , as this removes the eosin. The ribbons are now separated into sections. This is easily effected by a gentle stabbing motion with the point of the needle at the intersections.

Individual sections are now floated on to clean slides in the usual manner, and are allowed to drain by standing upright on filter paper. Blotting firmly at this stage is both expeditious and gives a flatter section.

When the sections are dry they are gently heated, placed in xylol to remove wax, and mounted in Canada balsam. 


\section{Conclusions}

The results obtained with this method compare favourably with methods previously used in this department. As early sections were under-stained with eosin, it was necessary to increase the staining time and to float section on to slides as soon as possible. There seems to be no reason why other staining techniques should not be adapted for use with this method, especially those in which precipitation of stain occurs, or where one of the reagents causes the section to float off the slide.

\section{Summary}

A method is given for which the apparatus used is readily available in almost all laboratories.

A set of fifty sections may be cut and stained by one technician in approximately two and a half hours, during which time there are quite long intervals when other work may be performed.

The use of alcohol for dehydration is obviated. The handling of single slides, a time-consuming operation, is cut to a minimum.

\section{REFERENCE}

Robb-Smith, A. H. T. (1937). J. Path. Bact., 46, 312.
Thanks are due to Professor R. J. V. Pulvertaft foro permission to publish this paper, and to Dr. A. D. Morgan for his interest and criticisms.

\section{Appendix}

1. Air bubbles in fluid are to be avoided if possible, $\frac{\overline{\bar{\rho}}}{\overline{5}}$ and should be removed from warm water by running $\widehat{\varnothing}$ fingers round the inside of the dish before floating on sections. Bubbles that are formed subsequently do not appear to cause uneven staining.

2. If a glass filter-pump is being used a wash-bottle is not needed when removing tap water or distilled $\vec{\omega}$ water, as these are obviously used fresh each time. $\%$ If a pump of the Hyvac type is used a wash-bottle is, of course, essential.

3. Harris's haematoxylin has been used, stainingi with which compares favourably with Ehrlich's, and it $\vec{A}$ has the advantage of being easily and rapidly pre- $-\overrightarrow{ }$ pared. Since it is ripened with mercuric oxide, it is 0 immediately ready for use. Glacial acetic acid (5 per cent) is added, to give more precise nuclear staining, and to neutralize any salts carried over from the tap water.

4. Differentiation may be controlled microscopic- $\vec{\varphi}$ ally in the usual manner by floating a single section 0 on to a clean slide. The acid alcohol is, of course, omitted if the progressive method of staining is preferred. 